VoL. 46 (1992) [287-294]

\title{
POINTS FIXES DANS LES ESPACES DES OPERATEURS NUCLEAIRES
}

\author{
Mourad Besbes
}

\begin{abstract}
We prove that some metric inequalities imply weak or weak-star normal structure. In particular, we prove that every $\omega^{*}$-compact convex set in the space $C_{1}\left(\ell^{p}, \ell^{q}\right)$ of nuclear operators from $\ell^{p}$ into $\ell^{q},(1<p, q<\infty, 1 / p+1 / q=1)$ has the weak* normal structure. This generalises a recent result of $C$. Lennard.
\end{abstract}

\section{INTRODUCTION}

Soient $p$ et $q$ deux réels dans $(1,+\infty)$ tels que $1 / p+1 / q=1$. On note $C_{\infty}\left(\ell^{q}, \ell^{p}\right)$ l'ensemble des opérateurs compacts de $\ell^{q}$ dans $\ell^{p}$ et $C_{1}\left(\ell^{p}, \ell^{q}\right)$ l'ensemble des opérateurs nucléaires de $\ell^{p}$ dans $\ell^{q}$. Il est alors classique d'identifier $C_{1}\left(\ell^{p}, \ell^{q}\right)$ et $\left[C_{\infty}\left(\ell^{q}, \ell^{p}\right)\right]^{*}$ (voir [13]). On notera $\omega^{*}$ la topologie associée à cette dualité.

\section{DÉFINITIONS:}

Soit $X$ un dual $X=Y^{*}$. On note $\omega^{*}$ la topologie préfaible $\sigma(X, Y)$. On dit que $X$ possède la propriété du point fixe pour les convexes $\omega^{*}$-compacts (on notera $\omega^{*}$.p.p.f cette propriété) si pour tout convexe $\omega^{*}$-compact $C$ et toute contraction

$$
T: C \rightarrow C ;\|T(x)-T(y)\| \leqslant\|x-y\| \forall x, y \in C,
$$

il existe $x_{0} \in C$, fixe par $T$.

Cette propriété est considérée par exemple dans [8] ou [12]. On sait [12] que $\ell^{1}=c_{0}^{*}$ possède la $\omega^{*}$.p.p.f.

Soit $C$ un convexe $\omega^{*}$-compact. On dira que $C$ possède la structure normale pour la topologie $\omega^{*}$ (On notera $\omega^{*} . S . N$. cette propriété) si pour tout sous convexe $K$ inclus dans $C, \omega^{*}$-fermé, non vide et non réduit à un point, il existe $x_{0} \in K$ non diametral. (c'est à dire, tel que $\sup _{y \in K}\left\|x_{0}-y\right\|<\operatorname{diam} K$ ). On définit de manière analogue la $\omega . S . N$ dans les convexes $\omega$-compacts.

Received 18th September, 1991.

L'auteur remercie G. Godefroy, P. Mazet et G. Pisier pour de fructueuses discussions.

Copyright Clearance Centre, Inc. Serial-fee code: 0004-9729/92 \$A2.00+0.00. 


\section{RESULTATS PRINCIPAUX}

Notre premier résultat étend un résultat obtenu par Lennard [10].

Theoreme 1.1. Tout convexe $\omega^{*}$-compact inclus dans $C_{1}\left(\ell^{p}, \ell^{q}\right)$ possède la $\omega^{*} . S . N$. En particulier $C_{1}\left(\ell^{p}, \ell^{q}\right)$ possède la $\omega^{*}$.p.p.f.

Démonstration: On établira d'abord deux lemmes techniques dont l'idée se trouve dans [1]. On désignera par projection naturelle définie sur $\ell^{r}$ une projection

$$
\text { définie par : } P_{n}(x)=\left(x_{1}, \ldots, x_{n}, 0 \ldots\right)
$$

si $x=\left(x_{1}, \ldots, x_{n}, x_{n+1}, \ldots\right) \in \ell^{r}, n$ étant un entier fixé.

Soient $P$ et $\widetilde{P}$ deux projections naturelles définies respectivement sur $\ell^{p}$ et $\ell^{q}$, et soient $Q$ et $\widetilde{Q}$ les projections définies par $P+Q=I$ et $\widetilde{P}+\widetilde{Q}=I$.

LEMME 1.2. Pour tout $x \in C_{1}\left(\ell^{p}, \ell^{q}\right)$, on a

$$
\|x\|_{C_{1}}^{q} \geqslant\left\|\tilde{P}_{x} P\right\|_{C_{1}}^{q}+\left\|\tilde{Q}_{x} P\right\|_{C_{1}}^{q}+\|x Q\|_{C_{1}}^{q} .
$$

DÉmonstration: Soit $y \in C_{\infty}\left(\ell^{q}, \ell^{p}\right)$

$$
\begin{aligned}
\|y\|_{C_{\infty}} & =\sup _{\xi \in \ell^{q},\|\xi\| \leqslant 1}\|y \xi\| \\
& =\sup _{\xi \in \ell^{q},\|\xi\| \leqslant 1}\left(\|P y \xi\|^{p}+\|Q y \xi\|^{p}\right)^{1 / p} \\
& \leqslant\left(\|P y\|_{C_{\infty}}^{p}+\|Q y\|_{C_{\infty}}^{p}\right)^{1 / p}
\end{aligned}
$$

De plus, on a:

$$
\|P y\|_{C_{\infty}}^{p} \leqslant\|P y \tilde{P}\|_{C_{\infty}}^{p}+\|P y \tilde{Q}\|_{C_{\infty}}^{p}
$$

en effet, pour $u \in \ell^{q}$, on a :

$$
\begin{aligned}
\|P y u\|^{p} & =\|P y(\widetilde{P} u+\widetilde{Q} u)\|^{p} \\
& =\|(P y \widetilde{P})(\widetilde{P} u)+(P y \tilde{Q})(\widetilde{Q} u)\|^{p} \\
& \leqslant(\|P y \tilde{P}\|\|\widetilde{P} u\|+\|P y \tilde{Q}\|\|\widetilde{Q} u\|)^{p} \\
& \leqslant\left(\|P y \tilde{P}\|^{p}+\left\|P_{y} \tilde{Q}\right\|^{p}\right)\left(\|\tilde{P} u\|^{q}+\|\widetilde{Q} u\|^{q}\right)^{p / q} .
\end{aligned}
$$

Il en résulte que

$$
\|y\|_{C_{\infty}}^{p} \leqslant\|P y \tilde{P}\|_{C_{\infty}}^{p}+\|P y \tilde{Q}\|_{C_{\infty}}^{p}+\|Q y\|_{C_{\infty}}^{p}
$$

et par dualité :

$$
\|x\|_{C_{1}}^{q} \geqslant\left\|\tilde{P}_{x} P\right\|_{C^{1}}^{q}+\left\|\tilde{Q}_{x} P\right\|_{C^{1}}^{q}+\|x Q\|_{C^{1}}^{q}
$$


LEMME 1.3. Soient $\left(x_{n}\right)_{n \geqslant 1}$ une suite d'éléments de $C_{1}\left(\ell^{p}, \ell^{q}\right)$ telle que $x_{n} \stackrel{\omega^{*}}{\longrightarrow}$ 0 et $x \in C_{1}\left(\ell^{p}, \ell^{q}\right)$ alors liminf $\left\|x_{n}+x\right\|^{q} \geqslant 1 / 2^{q / p} \liminf \left\|x_{n}\right\|^{q}+\|x\|^{q}$.

DÉMonstration: Comme $x_{n} \stackrel{\omega^{*}}{\longrightarrow} 0 \exists P_{n} \uparrow I$ et $\tilde{P}_{n} \uparrow I$ deux suites de projections naturelles définies respectivement sur $\ell^{p}$ et $\ell^{q}$ telles que :

$$
\left\|\tilde{P}_{n} x_{n} P_{n}\right\|_{n \rightarrow \infty} \rightarrow 0 \text { et }\left\|\tilde{P}_{n} x P_{n}-x\right\|_{n \rightarrow \infty} \rightarrow 0
$$

En utilisant le lemme précédent, on obtient :

$$
\left\|x_{n}+x\right\|^{q} \geqslant\left\|\tilde{P}_{n} x P_{n}\right\|^{q}+\left\|\tilde{Q}_{n} x_{n} P_{n}\right\|^{q}+\left\|x_{n} Q_{n}\right\|^{q}+\varepsilon_{n}
$$

avec $\lim _{n \rightarrow \infty} \varepsilon_{n}=0$ d'où

$$
\begin{array}{r}
\liminf \left\|x_{n}+x\right\|^{q} \geqslant\|x\|^{q}+\lim \inf \left(\left\|\widetilde{Q}_{n} x_{n} P_{n}\right\|^{q}+\left\|x_{n} Q_{n}\right\|^{q}\right) . \\
\text { Or } \quad \begin{aligned}
2^{1 / p}\left(\left\|\widetilde{Q}_{n} x_{n} P_{n}\right\|^{q}+\left\|x_{n} Q_{n}\right\|^{q}\right)^{1 / q} & \geqslant\left\|\widetilde{Q}_{n} x_{n} P_{n}\right\|+\left\|x_{n} Q_{n}\right\| \\
& \geqslant\left\|x_{n}\right\|-\left\|\widetilde{P}_{n} x_{n} P_{n}\right\|
\end{aligned}
\end{array}
$$

Donc: $\quad \liminf \left\|x_{n}+x\right\|^{q} \geqslant\|x\|^{q}+\frac{1}{2^{q / p}} \lim \inf \left\|x_{n}\right\|^{q}$.

REMARQues 1.4. Le coefficient $1 / 2^{q / p}$ dans le lemme 1.3 est nécessaire comme le montre l'exemple suivant. D'ailleurs dans le cas où $p=q=2$ le coefficient $1 / 2$ est le meilleur possible.

Exemple. Dans le cas où $p=q=2$ considérons les éléments $x_{n}$ et $x$ de $C_{1}=$ $C_{1}\left(\ell^{2}, \ell^{2}\right)$ définis par :

$$
x=\frac{1}{\alpha} e_{1} \otimes e_{1} \text { et } x_{n}=e_{1} \otimes e_{n}+e_{n} \otimes e_{1}+\alpha e_{n} \otimes e_{n}
$$

où $\alpha>0$ et $\left(e_{1}, \ldots, e_{n}, \ldots\right)$ est la base canonique de $\ell^{2}$. Il est alors facile de voir que $x_{n} \stackrel{\omega^{*}}{\longrightarrow} 0,\left\|x_{n}\right\|_{C_{1}}=\sqrt{\alpha^{2}+4}, \forall n \geqslant 1 ;\|x\|_{C_{1}}=1 / \alpha$ et $\left\|x_{n}+x\right\|_{C_{1}}=\left(\alpha^{2}+1\right) / \alpha$.

Ainsi si $C$ est un réel positif vérifiant l'inégalité

$$
\begin{aligned}
& \lim \inf \left\|x_{n}+x\right\|^{2} \geqslant C \lim \inf \left\|x_{n}\right\|^{2}+\|x\|^{2}, \\
& \text { alors } {\left[\frac{\alpha^{2}+1}{\alpha}\right]^{2} \geqslant C\left(\alpha^{2}+4\right)+\frac{1}{\alpha^{2}}, \forall \alpha>0 }
\end{aligned}
$$


et donc $C \leqslant\left(\alpha^{2}+2\right) /\left(\alpha^{2}+4\right)$, pour tout $\alpha>0$, ce qui entraine que $C \leqslant 1 / 2$.

En prenant $\alpha=1$, on a : liminf $\left\|x_{n}\right\|>\liminf \left\|x_{n}+x\right\|$.

Cet exemple montre donc aussi que l'espace $C_{1}\left(\ell^{2}\right)$ ne vérifie pas la condition d'Opial dont il sera question à la fin de l'article.

Remarquons que le lemme 1.3 permet de montrer aussi que l'espace $C_{1}\left(\ell^{p}, \ell^{q}\right)$ vérifie la propriété $U K K^{*}[6]$ définie par : $\forall \varepsilon>0 \quad \exists \delta>0$ tel que

$$
\left.\begin{array}{l}
\left\|x_{n}\right\| \leqslant 1 \\
x_{n} \stackrel{\omega^{*}}{\longrightarrow} x \\
\inf \left\{\left\|x_{n}-x_{m}\right\|, n \neq m\right\} \geqslant \varepsilon
\end{array}\right\} \Rightarrow\|x\| \leqslant 1-\delta .
$$

En effet, considérons une suite $\left(x_{n}\right)$ telle que :

$$
\left\|x_{n}\right\| \leqslant 1, x_{n} \stackrel{\omega^{*}}{\longrightarrow} x \text { et } \inf \left\{\left\|x_{n}-x_{m}\right\|, n \neq m\right\} \geqslant \varepsilon
$$

alors $\liminf \left\|x_{n}-x\right\| \geqslant \varepsilon / 2$.

D'après le lemme 1.2, on obtient,

et donc

$$
\begin{aligned}
& \|x\|^{q}+\frac{1}{2^{q} .2^{q / p}} \varepsilon^{q} \leqslant 1 \\
& \|x\| \leqslant\left(1-\frac{\varepsilon^{q}}{2^{(q / p+q)}}\right)^{1 / q}
\end{aligned}
$$

La fin de la démonstration du théorème 1.1 est maintenant classique [12, par exemple].

Soit $C$ un convexe $\omega^{*}$-compact, non vide et non réduit à un point où tout point est diamétral. Il existe alors une suite $\left(x_{n}\right)_{n \geqslant 1}$ dans $C$ telle que :

$$
\forall n \neq m,\left\|x_{n}-x_{m}\right\| \geqslant \frac{\delta}{2}
$$

où $\delta=\operatorname{diam} K$.

Quitte à en extraire une sous-suite et à faire une translation, on peut supposer que $\boldsymbol{x}_{n} \stackrel{\boldsymbol{\omega}^{*}}{\longrightarrow} 0$. D'après $\left(^{*}\right)$, on a $\liminf \left\|\boldsymbol{x}_{n}\right\|>0$.

D'autre part, d'après le lemme 1.3 on $a$ :

$$
\forall x \in K \quad \liminf \left\|x_{n}-x\right\|^{q} \geqslant \frac{1}{2^{q / p}} \liminf \left\|x_{n}\right\|^{q}+\|x\|^{q}
$$

et donc $\sup _{x \in K}\|x\|<\operatorname{diam} K$, ce qui contredit l'hypothèse. 


\section{Generalisation des resultats}

On se propose maintenant de généraliser les résultats précédents.

Proposition 2.1. Soit $X$ un Banach. Supposons qu'il existe $p>0, \alpha_{p}$ et $\beta_{p} \in[0,1]$ tels que

(i) $\alpha_{p}+\beta_{p}>1$

(ii) $\sup _{n}\left\|x_{n}-x\right\|^{p} \geqslant \alpha_{p}\|x\|^{p}+\beta_{p} \liminf \left\|x_{n}\right\|^{p}$

pour toute suite $\left(x_{n}\right)$ telle que $x_{n} \stackrel{\omega}{\longrightarrow} 0$ et tout $x \in X$. Alors tout convexe $\omega$-compact de $X$ possède la $\boldsymbol{\omega} . S . N$.

DÉmonstration: Supposons qu'il existe un convexe $K \omega$-compact dans $X$, non vide et non réduit à un point où tout point est diamétral. D'après [4], il existe une suite $\left(x_{n}\right)_{n \geqslant 1}$ dans $K$ telle que : $\lim _{n \rightarrow \infty} d\left(x_{n+1}, \operatorname{conv}\left(x_{1}, \ldots, x_{n}\right)\right)=\delta\left(^{*}\right)$ ( $\delta$ étant le diamètre de $K$ ).

Quitte à en entraire une sous-suite et à faire une translation on peut supposer que $x_{n} \stackrel{\omega}{\longrightarrow} 0 \in K$.

D'après $\left(^{*}\right)$ on a $\liminf _{n \rightarrow \infty}\left\|x_{n}\right\|=\delta$ car $0 \in \overline{\operatorname{conv}\left(x_{1}, \ldots, x_{n}, \ldots\right)}$. En appliquant l'hy pothèse de la proposition à la suite $\left(x_{n}\right)_{n \geqslant 1}$, on obtient $\delta^{p} \geqslant \alpha_{p}\|x\|^{p}+\beta_{p} \delta^{p} \forall x \in K$ ce qui montre que 0 est non diamétral dans $K$. Il en résulte que tout convexe $\omega$-compact de $X$ possède la $\boldsymbol{\omega} . S . N$.

Dans l'argument ci-dessus, on utilise le théorème de Mazur sur la compatibilité des topologies faibles et fortes pour démontrer que $0 \in \overline{\text { conv }}\left(x_{1}, \ldots, x_{n}, \ldots\right)$. Dans le cas où $X$ est un dual séparable, on peut modifier cet argument grâce à une construction dûe à Lennard [11] ce qui permet d'obtenir la Proposition 2.2:

Proposition 2.2. Soit $X$ un dual séparable. Supposons qu'il existe $p>0, \alpha_{p}$ et $\beta_{p} \in[0,1]$ tels que :

(i) $\alpha_{p}+\beta_{p}>1$,

(ii) $\sup _{n}\left\|x_{n}-x\right\|^{p} \geqslant \alpha_{p}\|x\|^{p}+\beta_{p} \liminf \left\|x_{n}\right\|^{p}$

pour toute suite $\left(x_{n}\right)$ telle que $x_{n} \stackrel{\omega^{*}}{\longrightarrow} 0$ et tout $x \in X$. Alors tout convexe $\omega^{*}$-compact de $X$ possède la $\omega^{*} . S . N$.

DÉmonstration: Supposons qu'il existe un convexe $K \omega^{*}$-compact, inclus dans $X$, non vide et non réduit à un point où tout point est diamétral. Soit $\left(u_{n}\right)$ une suite dense dans $K$ pour la topologie forte. En suivant [11], on construit une suite $\left(x_{n}\right)$ d'éléments de $K$ telle que:

$$
\forall n \geqslant 1, d\left(x_{n+1}, \operatorname{conv}\left(x_{1}, \ldots, x_{n}, u_{1}, \ldots, u_{n}\right)\right) \geqslant \delta\left(1-\frac{1}{n}\right)
$$


(où $\delta$ est le diamètre de $K$ ). Comme $K$ est $\omega^{*}$-compact et $X$ est séparable, il existe une sous suite $\left(x_{\varphi(n)}\right)$ telle que $x_{\varphi(n)} \stackrel{\omega^{*}}{\longrightarrow} x \in K$. De plus, comme la suite $\left(u_{n}\right)$ est dense dans $K$, il existe une sous suite $\left(x_{\varphi \circ \psi(n)}\right)$ telle que $\lim _{n \rightarrow \infty}\left\|x_{\varphi \circ \psi(n)}-x\right\|=\delta$. (On peut construire cette sous suite par récurrence en choisissant pour chaque $n$ un élément $u_{\delta(n)}$ tel que $\left\|u_{\delta(n)}-x\right\|<1 / n$ et $\psi(n)$ tel que $\psi(n)>\psi(n-1)$ et $\left.\varphi o \psi(n)>\delta(n)\right)$.

En appliquant l'hypothèse de la proposition à la suite $\left(x_{\varphi \circ \psi(n)}-x\right)$, on montre comme ci-dessus que $x$ est non diamétral dans $K$. Il en résulte que tout convexe $\omega^{*}$-compact de $X$ possède la $\omega^{*} . S . N$.

Les propositions ci-dessus permettent de montrer des résultats d'existence de points fixes dans les espaces isomorphes à $C_{1}\left(\ell^{p}, \ell^{q}\right)$. Nous avons en effet le:

Corollatre 2.3. Soit $X$ un Banach $K$-isomorphe à $C_{1}\left(\ell^{p}, \ell^{q}\right)$; supposons que $K<\left(1+1 /\left(2^{q / p}\right)\right)^{1 / q}$; alors tout convexe $\omega$-compact de $X$ possède la w.S.N.

REMARQUeS 2.4. La proposition 2.1 permet en particulier de retrouver les résultats suivants (voir [9]):

(a) Soit $X$ un Banach $K$-isomorphe à $\ell^{p}$ avec $1 \leqslant p<\infty$ et $K<2^{1 / p}$. Alors tout convexe $\omega$-compact de $X$ possède la $\omega . S . N$.

(b) Cas des espaces de James: Soit

$$
J_{p}=\left\{\left(x_{n}\right) \in c_{0} ; \sup \sum_{k=1}^{K}\left|x_{n_{2 k}}-x_{n_{2 k-1}}\right|^{p}<\infty, n_{1}<n_{2}<\cdots<n_{2 K}, K>0\right\} .
$$

On munit l'espace $J_{p}$ de la norme :

$$
\|x\|=\sup \left(\sum_{k=1}^{K}\left|x_{n_{2 k}}-x_{n_{2 k-1}}\right|^{p}\right)^{1 / p}
$$

$J_{p}$ est alors un espace de Banach pour tout $p \in\left[1,+\infty\left[\right.\right.$. Si $X$ est $K$-isomorphe à $J_{p}$ avec $K<2^{1 / p}$, alors tout convexe $\omega$-compact de $X$ a la $\omega . S . N$.

REMARQUE 2.5. Examinons les deux cas particuliers suivants de la proposition 2.2:

(a) $\alpha_{p}=1$ et $\beta_{p}>0$

(b) $\beta_{p}=1$ et $\alpha_{p}>0$

Dans le premier cas, on peut montrer comme ci-dessus pour $C_{1}\left(\ell^{p}, \ell^{q}\right)$ que $X$ vérifie la propriété $U . K . K .^{*}$ Dans le deuxième cas, l'espace $X$ vérifie la propriété d'Opial pour la topologie $\omega^{*}$ définie par:

On dit que $X$ vérifie la condition d'Opial [16], pour la topologie $\omega^{*}$ si pour toute suite $\left(x_{n}\right)$ dans $X$ telle que $x_{n} \stackrel{\omega^{*}}{\longrightarrow} x_{0}$ et tout $x \neq x_{0}$ on a:

$$
\liminf \left\|x_{n}-x\right\|>\liminf \left\|x_{n}-x_{0}\right\| \text {. }
$$


REMARQUE 2.6.

* Tout dual vérifiant la condition d'Opial pour la topologie $\omega^{*}$ possède la $\omega^{*}$.p.p.f (voir [5] ou [16]).

* L'espace $\ell^{1}=c_{0}^{*}$ vérifie la condition d'Opial pour $\sigma\left(\ell^{1}, c_{0}\right)$. Il en est de même des espaces $\ell^{p}, 1<p<\infty$ pour $\sigma\left(\ell^{p}, \ell^{q}\right)$ où $1 / p+1 / q=1$.

* L'exemple donné dans la remarque 1.4 montre que $C_{1}$ ne vérifie pas la condition d'Opial.

* L'exemple suivant montre que l'espace de Hardy $H^{1}$, ne vérifie pas la condition d'Opial pour la topologie préfaible naturelle. Néanmoins, on montre ([2] ou [3]) que $H^{1}$ a la $\omega^{*}$.p.p.f.

Posons $f_{n}(z)=1 / 2 z^{2 n}+z^{n}$ et $f(z)=1 / 2$

alors

$$
\begin{aligned}
f_{n} \stackrel{\omega^{*}}{\longrightarrow} 0,\left\|f_{n}\right\|_{H^{1}} & =\int_{0}^{2 \pi}\left|1+\frac{1}{2} e^{i n \theta}\right| \frac{d \theta}{2 \pi}>1 \\
\left\|f_{n}+f\right\|_{H^{1}} & =\int_{0}^{2 \pi}\left|\frac{1}{2}+e^{i n \theta}+\frac{1}{2} e^{2 i n \theta}\right| \frac{d \theta}{2 \pi} \\
& =\int_{0}^{2 \pi}(1+\cos n \theta) \frac{d \theta}{2 \pi}=1
\end{aligned}
$$

* D. Van Dulst a démontré dans [5] que pour tout dual séparable $X$ on peut trouver une norme duale || , équivalente à sa norme initiale, telle que $(X,||)$ vérifie la condition d'Opial.

Notre dernier résultat fournit une réponse positive à une question de Sims [14] (voir aussi [7] et [15]) dans le cas où $X$ est séparable.

Theoreme 2.7. Soit $X$ un dual séparable vérifiant la condition d'Opial pour la topologie $\omega^{*}$. Alors tout convexe $\omega^{*}$-compact de $X$ possède la $\omega^{*} . S . N$.

\section{REFERENCES}

[1] A. Arazy, 'More on convergence in unitary matrix spaces', Proc. Amer. Math. Soc. . 83 (1981), 44-48.

[2] M. Besbes, 'Points fixes des contractions définies sur un convexe $L^{\circ}$-fermé de $L^{1}$ ', C.R.A. Sciences de Paris, t. 311 (1990), 243-246.

[3] M. Besbes, S. Dilworth, P. Dowling and C. Lennard, 'New convexity and fixed point properties in Hardy and Lebesgue-Bochner spaces', J. Funct. Anal. (to appear).

[4] M.S. Brodski and D.P. Milman, 'On the center of a convex set', Dokl. Acad. Nauk. USSR 59 (1948)), 837-840.

[5] D. Van Dulst, 'Equivalent norms and the fixed point property for non-expansive mappings', J. London Math. Soc. 25 (1982), 139-144. 
[6] D. Van Dulst and B. Sims, 'Fixed points of non-expansive mappings and Chebyshev centers in Banach spaces with norms of type (KK)', in Lecture notes in Math. 991, pp. 34-43 (Springer-Verlag, Berlin, Heidelberg, New York, 1983).

[7] J.P. Gossez and E. Lami Dozo, 'Normal structure and Schauder bases', Acad. Roy. Belg. Bull. Cl. Sci. 55 (1969), 673-681.

[8] A. Khamsi, Thèse de doctorat (Université Paris VI, 1987).

[9] A. Khamsi, 'Normal structure for Banach spaces with Schauder decomposition', Canad. Math. Bull. 32 (1989), 344-351.

[10] C. Lennard, ' $C_{1}$ is uniformly Kadec Klee*', Proc. Amer. Math. Soc. 109 (1990), 71-77.

[11] C. Lennard, 'A new convexity property that implies a fixed point property for $L^{1}$, (préprint).

[12] T.C. Lim, 'Asymptotic centers and non-expansive mappings in conjugate Banach spaces', Pacific J. Math. 90 (1980), 135-143.

[13] B. Simon, 'Convergence in trace ideals', Proc. Amer. Math. Soc. 83 (1981), 39-43.

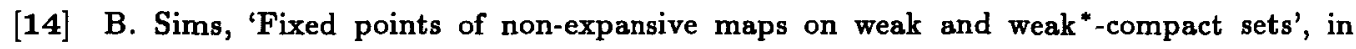
Queen's University of Kingston. Lecture Notes, 1982.

[15] S. Swaminathan, 'Normal structure in Banach spaces and its generalisations', Contemp. Math. A.M.S. Providence 18, 201-215.

[16] Z. Opial, 'Weak convergence of the sequence of successive approximations for non-expansive mappings', Bull. Amer. Math. Soc. 73 (1967), 591-597.

Equipe d'analyse, Boîte 186

Université Paris VI

4, Place Jussieu

75252 - Paris Cedex 05

France 